\title{
INTEGRAL REPRESENTATION OF MULTIPLICATIVE, INVOLUTION PRESERVING OPERATORS IN

$$
\mathscr{L}(C(S), E)^{1}
$$

\section{GERALD W. JOHNSON}

1. Introduction. Throughout, $S$ will be a compact Hausdorff space and $E$ will be a Banach space which is the dual of another Banach space $F . C(S)$ will denote the space of complex-valued continuous functions on $S$ topologized with the topology of uniform convergence. $\mathscr{L}(C(S), E)$ will denote the space of continuous linear operators from $C(S)$ to $E$. A theorem of Gil de Lamadrid [5, p. 103] identifies $\mathfrak{L}(C(S), E)$ with a space of $E$-valued "measures," the correspondence between operator and measure being given by integration. A closely related result was given earlier by Bartle, Dunford, and Schwartz [2]. Now if $E$ is a Banach algebra with involution [7, p. 178], it makes sense to consider operators which are not only continuous and linear but which also preserve multiplication and involution. A natural question arises: How are these additional properties reflected in the representing measure? We answer this question under additional restrictions on $E$. We also give several examples of spaces $E$ satisfying the hypotheses of our theorems. One can use the results of this paper to prove the Spectral Theorem for bounded operators; but the proof follows standard lines and will not be included (see [6, p. 99]).

We conclude this introduction with a precise description of Gil de Lamadrid's Theorem. Our description differs from Gil de Lamadrid's, but it is not difficult to verify that they are equivalent. We consider the class $N(S, E)$ of set functions $m$ from $B(S)$, the Borel class of $S$, to $E$ which are countably additive and regular with respect to the weak topology $\sigma(E, F)$ on $E$ induced by $F$. To say that $m: \AA(S) \rightarrow E$ is regular with respect to the topology $\sigma(E, F)$ means that, for every $B \in \Theta(S)$ and every $\sigma(E, F)$-neighborhood $N$ of 0 , there exists a compact set $K$ and an open set $U$ such that $K \subseteq B \subseteq U$ and, if $A \subseteq U-K$, then $m(A) \in N$. Defining addition and scalar-multiplication in $N(S, E)$ in the usual set-wise fashion; i.e., $\left(m_{1}+m_{2}\right)(B)=m_{1}(B)$ $+m_{2}(B)$ and $\left(\alpha m_{1}\right)(B)=\alpha m_{1}(B), N(S, E)$ is a vector space. The following formula defines a norm on $N(S, E)$ making it a Banach space:

Received by the editors December 7, 1968.

1 This paper is based on Chapter 5 of the author's Ph.D. Thesis [6] written at the University of Minnesota under the direction of Professor Gil de Lamadrid. The work was supported in part by the National Science Foundation. 
$\|m\|=\sup \left\{\left\|\sum_{j=1}^{n} \alpha_{j} m\left(B_{j}\right)\right\|\right\}$ where $B_{1}, \cdots, B_{n}$ is a partition of $S$ by elements of $B(S)$ and each $\alpha_{j} \in C$, the field of complex numbers, satisfying $\left|\alpha_{j}\right| \leqq 1$. Now given $m \in N(S, E)$ we define $L: C(S) \rightarrow E$ by the formula: $L(f)=\int_{S} f d m$ where the integration may be interpreted via [1]. Gil de Lamadrid's Theorem says that this correspondence sets up an isometric isomorphism of $N(S, E)$ onto $\mathfrak{L}(C(S), E)$.

2. The representation theorems. Our first theorem deals with multiplicative operators. The proof is similar in outline to the proof of the Spectral Theorem as in [4, circa p. 897].

Theorem 1. Let E be a Banach algebra which, as a Banach space, is the dual of another Banach space F; further suppose that the multiplication in $E$ is separately continuous with respect to the topology $\sigma(E, F)$. Let $L \in \mathfrak{L}(C(S), E)$ and let $m \in N(S, E)$ be its representing measure. $L$ satisfies $L(f g)=L(f) L(g)$ for all $f, g \in C(S)$ if and only if $m\left(B_{1} \cap B_{2}\right)$ $=m\left(B_{1}\right) m\left(B_{2}\right)$ for all $B_{1}, B_{2} \in ß(S)$.

PRoOf. We begin by giving some useful facts in integration theory. Let $m \in N(S, E)$ and let $e \in E$. We define $m_{e}: B(S) \rightarrow E$ by $m_{e}(B)$ $=m(B) e$, and we let $m^{e}: B(S) \rightarrow E$ be defined by $m^{e}(B)=e m(B)$. Since multiplication in $E$ is separately continuous with respect to the topology $\sigma(E, F)$, we easily see that $m_{e}$ and $m^{e}$ are in $N(S, E)$. For $f \in C(S)$, we get $\int_{S} f d m_{e}=\left(\int_{S} f d m\right) e$ and $\int_{S} f d m^{e}=e\left(\int_{S} f d m\right)$. These formulas are clear for $f$ a simple function and are obtained for $f \in C(S)$ by passage to the limit (see $[1$, p. 341$]$ ).

Now let $B_{0} \in B(S)$ and define $m_{B_{0}}: B(S) \rightarrow E$ by $m_{B_{0}}(B)=m\left(B \cap B_{0}\right)$. $m_{B_{0}} \in N(S, E)$ and we get $\int_{S} f d m_{B_{0}}=\int_{B_{0}} f d m, f \in C(S)$.

We need one additional fact from integration theory. If $g \in C(S)$, we define $m_{g}: B(S) \rightarrow E$ by $m_{g}(B)=\int_{B} g d m$. We claim that $m_{g} \in N(S, E)$ and that, for $f \in C(S), \int_{S} f d m_{g}=\int_{S} f g d m$.

Let $M(S)$ be the set of countably additive, complex-valued, regular Borel measures on $S . N(S, E)$ may alternately be described as the class of set functions $m: B(S) \rightarrow E$ such that $m(\cdot)(x) \in M(S)$ for all $x \in F$. Let $x \in F$. It is not difficult to establish that $m_{g}(B)(x)$ $=\int_{B} \operatorname{gd} m(\cdot)(x)$. Hence $m_{\theta}(\cdot)(x)$ is the indefinite integral of $m(\cdot)(x)$ $\in M(S)$. Such an indefinite integral is countably additive [3, p. 152]. $m_{g}(\cdot)(x)$ must be regular since $m(\cdot)(x)$ is regular. This follows from $\left[3\right.$, p. 114]. Thus $m_{\theta} \in N(S, E)$.

To establish the formula, it suffices to show that $\left(\int_{S} f d m_{g}\right)(x)$ $=\left(\int_{S} f g d m\right)(x)$ for all $x \in F$; that is, it suffices to show $\int_{S} f d m_{g}(\cdot)(x)$ $=\int_{S} f g d m(\cdot)(x)$ for $x \in F$. But this formula is given for complex measures in [3, p. 180]. 
Now suppose $L(f g)=L(f) L(g)$ for all $f, g \in C(S)$ and let $B_{1}, B_{2}$ $\in B(S)$. We wish to show that $m\left(B_{1} \cap B_{2}\right)=m\left(B_{1}\right) m\left(B_{2}\right)$. It suffices to show that $m_{B_{2}}=m_{m\left(B_{2}\right)}$. Let $g \in C(S)$. Since the Gil de Lamadrid Theorem gives a one-to-one correspondence between $\mathfrak{L}(C(S), E)$ and $N(S, E)$, it suffices to show that $\int_{S} g d m_{B_{2}}=\int_{S} g d m_{m\left(B_{2}\right)}$. Now $\int_{S} g d m_{B_{2}}$ $=\int_{B_{2}} g d m=m_{g}\left(B_{2}\right)$. Also $\int_{S} g d m_{m\left(B_{2}\right)}=\left(\int_{S} g d m\right) m\left(B_{2}\right)=L(g) m\left(B_{2}\right)$. Thus to show $\int_{S} g d m_{B_{2}}=\int_{S} g d m_{m\left(B_{2}\right)}$, it suffices to show that $m_{g}=m^{L(\theta)}$. Let $f \in C(S)$. It suffices to show that $\int_{S} f d m_{g}=\int_{S} f d m^{L(\theta)}$. However, $\int_{S} f d m_{g}=\int_{S} f g d m=L(f g)=L(g) L(f)=L(g) \int_{S} f d m$ $=\int_{S} f d m^{L(0)} \cdot m\left(B_{1} \cap B_{2}\right)=m\left(B_{1}\right) m\left(B_{2}\right)$ now follows.

The converse is easier to establish; we outline the argument. Suppose $m \in N(S, E)$ satisfying $m\left(B_{1} \cap B_{2}\right)=m\left(B_{1}\right) m\left(B_{2}\right)$ for all $B_{1}, B_{2}$ $\in \Re(S)$ and let $L$ be the corresponding operator. Let $f, g \in C(S)$. We must establish the formula $\int_{S} f g d m=\left(\int_{S} f d m\right)\left(\int_{S} g d m\right)$. Let $f=\chi_{B}$ be the characteristic function of $B \in B(S)$. When $g$ is also the characteristic function of a set in $B(S)$, the formula is obvious. The formula follows from the linearity of the integral $[1, \mathrm{p} .342]$ when $g$ is a simple function. For $g \in C(S)$, the formula follows by passage to the limit. We now have the formula for $f=\chi_{B}$ and $g \in C(S)$. The case where $f$ is a simple function follows by linearity. Finally, by using Bartle's Bounded Convergence Theorem [1, p. 345] and passing to the limit, we get the result for arbitrary $f \in C(S)$. This completes the proof.

Our second theorem deals with involution preserving members of $\mathfrak{L}(C(S), E)$. If $f \in C(S), f^{*}$ will denote the complex conjugate of $f$. The map $f \rightarrow f^{*}$ is an involution on $C(S)$ and, in fact, $C(S)$ is a Banach algebra with isometric involution.

THEOREM 2. Let E be a Banach algebra with isometric involution*. Suppose that E, as a Banach space, is the dual of another Banach space $F$. Finally, suppose that the involution is continuous with respect to the topology $\sigma(E, F)$. Let $L \in \mathfrak{L}(C(S), E)$ and let $m$ be its representing measure. $L$ satisfies $L\left(f^{*}\right)=L(f)^{*}$ for all $f \in C(S)$ if and only if $m(B)$ $=m(B) *$ for all $B \in B(S)$.

Proof. Let $m \in N(S, E)$. We claim that the set function $m^{*}: B(S) \rightarrow E$ defined by $m^{*}(B)=m(B) *$ is in $N(S, E)$. Let $x \in F$. It suffices to show that $m^{*}(\cdot)(x) \in M(S)$. Define $x_{0}: E \rightarrow C$ by the equation $x_{0}(e)$ $=x\left(e^{*}\right)^{-}$where the bar denotes the conjugate of the complex number $x\left(e^{*}\right) . x_{0}$ is linear and, because of the assumption that ${ }^{*}$ is continuous with respect to the topology $\sigma(E, F)$, and because the map $e \rightarrow x\left(e^{*}\right)^{-}$ is composed of the maps $e \rightarrow e^{*} \rightarrow x\left(e^{*}\right) \rightarrow x\left(e^{*}\right)^{-}$, we see that $x_{0}$ is a continuous linear functional on $(E, \sigma(E, F))$. Hence $x_{0}$ may be regarded as an element of $F\left[9\right.$, p. 124]. Now $m(B)\left(x_{0}\right)^{-}=m(B)^{*}(x)^{--}$ 
$=m^{*}(B)(x)$. But $m(\cdot)\left(x_{0}\right) \in M(S)$ and so $m(\cdot)\left(x_{0}\right)-\in M(S)$ and hence $m^{*}(\cdot)(x) \in M(S)$.

Next we observe that the formula $\left(\int_{S} f d m\right)^{*}=\int_{S} f^{*} d m^{*}$ is valid for $f \in C(S)$ and $m \in N(S, E)$. This is established in the usual way.

Now suppose $L\left(f^{*}\right)=L(f)^{*}$ for all $f \in C(S)$ and let $m$ be the representing measure for $L$. Now $\int_{S} f^{*} d m=\left(\int_{S} f d m\right)^{*}=\int_{S} f^{*} d m^{*}$. Since this holds for all $f \in C(S)$ and since $m, m^{*} \in N(S, E)$, Gil de Lamadrid's Theorem implies that $m=m^{*}$.

Conversely, suppose $m=m^{*}$; then $\left(\int_{S} f d m\right)^{*}=\int_{S} f^{*} d m^{*}=\int_{S} f^{*} d m$. Thus $L(f)^{*}=L\left(f^{*}\right)$. This completes the proof.

Next we list some Banach algebras to which Theorems 1 and 2 may be applied: (a) If $X$ is a reflexive Banach space, $\mathscr{L}(X)$ satisfies the hypotheses of Theorem 1 . We mention that $\mathscr{L}(X)$ is the dual of $X \otimes_{\gamma} X^{\prime}[11$, p. 47]. (b) Both Theorems 1 and 2 are applicable to any $B^{*}$-algebra $[9$, p. 180] which, as a Banach space, is the dual of another Banach space. These are exactly the $B^{*}$-algebras identifiable with $W^{*}$-algebras ([8] and [12]). $\&(H)$ is such a $B^{*}$-algebra where $H$ is a Hilbert space If $(S, \Sigma, \mu)$ is a measure space such that $L_{1}^{\prime}(S, \Sigma, \mu)$ $=L_{\infty}(S, \Sigma, \mu)$ (see $\left[3\right.$, p. 289]), then $L_{\infty}(S, \Sigma, \mu)$ is another such $B^{*}$ algebra. (c) Theorems 1 and 2 apply to any $H^{*}$-algebra [7, p. 272]. In particular they apply to $L_{2}(G)$ for any compact group $G$ where convolution is taken as multiplication [7, p. 330]. Also they apply to the Schmidt class of operators on a Hilbert space ([7, pp. 285-288] and [10, pp. 29-36]). (d) Let $G$ be a locally compact topological group. With convolution as multiplication, $M(G)$ satisfies the hypotheses of both theorems. (e) The trace class of operators on a Hilbert space $[10$, p. 37] also satisfies the hypotheses of both theorems.

\section{BiBLIOGRAPHY}

1. R. G. Bartle, A general bilinear vector integral, Studia Math. 15 (1956), 337-352.

2. R. G. Bartle, N. Dunford and J. Schwartz, Weak compactness and vector measures, Canad. J. Math. 7 (1955), 289-305.

3. N. Dunford and J. Schwartz, Linear operators. Part I: General theory, Interscience, New York and London, 1958.

4. - Linear operators. Part II: Spectral theory self-adjoint operators in hilbert space, Interscience, New York and London, 1964.

5. J. Gil de Lamadrid, Measures and tensors, Trans. Amer. Math. Soc. 114 (1965), 98-121.

6. Gerald W. Johnson, Integral representations of certain linear operators, Thesis, University of Minnesota, Minneapolis, Minn., 1968.

7. C. E. Rickart, General theory of banach algebras, Van Nostrand, Princeton, N. J., 1960.

8. S. Sakai, A characterization of $W^{*}$-algebras, Pacific J. Math. 6(1956), 763-773. 
9. H. H. Schaefer, Topological vector spaces, Macmillan, New York, 1966.

10. R. Schatten, Norm ideals of completely continuous operators, Ergebnisse der Mathematik und ihrer Grenzgebeite, Heft 27, Springer-Verlag, Berlin, 1960.

11. - A theory of cross-spaces, Ann. of Math. Studies, no. 26, Princeton Univ. Press, Princeton, N. J., 1950.

12. P. C. Shields, A new topology for von Neumann algebras, Bull. Amer. Math. Soc. 65 (1959), 267-269.

UNIVERSITY OF NEBRASKA 